\title{
REVIEW
}

Open Access

\section{Clinical considerations for medication- related osteonecrosis of the jaw: a comprehensive literature review}

\author{
Mampei Kawahara ${ }^{1 \dagger}$, Shinichiro Kuroshima ${ }^{1,2^{*}+}$ (D) and Takashi Sawase ${ }^{1,2}$
}

\begin{abstract}
Background: Medication-related osteonecrosis of the jaw (MRONJ), which was first reported as bisphosphonaterelated osteonecrosis of the jaw (BRONJ) in bisphosphonate users, is a rare but severe soft and hard tissue disease induced by several types of medications. There has been a deluge of information about MRONJ, such as epidemiology, risk factors, clinical recommendations for dental treatment to prevent it, and treatment strategies in medication-prescribed users. The aim of this study was to comprehensively review recent articles and provide the current scientific information about MRONJ, especially clinical considerations or recommendations for dental treatment to prevent its occurrence.
\end{abstract}

Materials and methods: The current literature review was mainly based on 14 systematic reviews with or without meta-analysis, 4 position papers, 1 consensus statement, 1 clinical guideline, and 2 clinical reviews regarding MRONJ after a PubMed database and manual searches according to inclusion and exclusion criteria. Moreover, 53 articles were selected by manual search in regard to all references from selected articles and other articles identified on the PubMed search, irrespective of publication date, and inclusion and exclusion criteria.

Results: The incidence and prevalence of MRONJ are relatively low, although they are clearly higher in cancer patients receiving high-dose antiresorptive agents or angiogenesis inhibitors rather than osteoporosis patients receiving oral bisphosphonates or denosumab. There are many types of local, systemic, and other risk factors for the development of MRONJ. Clinical recommendations are provided for each clinical situation of patients to prevent MRONJ. There are also treatment strategies for MRONJ in each stage.

(Continued on next page)

\footnotetext{
* Correspondence: kuroshima@nagasaki-u.ac.jp

${ }^{\dagger}$ Mampei Kawahara and Shinichiro Kuroshima contributed equally to this work.

'Oral \& Maxillofacial Implant Center, Nagasaki University Hospital, 852-8588, Nagasaki, Japan

2Department of Applied Prosthodontics, Graduate School of Biomedical Sciences, Nagasaki University, Nagasaki 852-8588, Japan
}

\section{Springer Open}

(c) The Author(s). 2021 Open Access This article is licensed under a Creative Commons Attribution 4.0 International License, which permits use, sharing, adaptation, distribution and reproduction in any medium or format, as long as you give appropriate credit to the original author(s) and the source, provide a link to the Creative Commons licence, and indicate if changes were made. The images or other third party material in this article are included in the article's Creative Commons licence, unless indicated otherwise in a credit line to the material. If material is not included in the article's Creative Commons licence and your intended use is not permitted by statutory regulation or exceeds the permitted use, you will need to obtain permission directly from the copyright holder. To view a copy of this licence, visit http://creativecommons.org/licenses/by/4.0/. 
(Continued from previous page)

Conclusions: General dentists should perform appropriate dental treatment to prevent MRONJ in the patients prior to or when receiving medications that could induce MRONJ. Moreover, there are treatment strategies for MRONJ in each stage that oral surgeons could follow. Adequate and updated clinical information regarding MRONJ based on scientific data is required whenever possible.

Keywords: Medication-related osteonecrosis of the jaw, Antiresorptive agents, Bisphosphonates, Denosumab, Angiogenesis inhibitors

\section{Background}

Medication-related osteonecrosis of the jaw (MRONJ) $[1,2]$, which includes bisphosphonate-related osteonecrosis of the jaw (BRONJ) [3], denosumab-related osteonecrosis of the jaw (DRONJ) $[4,5]$, and osteonecrosis of the jaw induced by angiogenesis inhibitors [6], is a rare but intractable disease. MRONJ has been shown to be one of the serious adverse side effects of antiresorptive agents such as bisphosphonates and anti-receptor activator of NF-kappa B ligand (denosumab) or angiogenesis inhibitors. Antiresorptive agents have been widely used to treat osteoporosis, hypercalcemia caused by malignant tumors, and skeletal-related events (SREs) such as bone pain and pathological fractures of vertebrae induced by multiple myeloma and solid tumors. Angiogenesis inhibitors such as the anti-vascular endothelial growth factor (VEGF) antibody agent bevacizumab have also been used for the treatment of several cancers. Recently, other medications such as neutralizing antibodies to tumor necrosis factor (TNF)- $\alpha, \mathrm{CD} 20$, and sclerostin, and molecular targeted drugs, have been reported to possibly induce MRONJ [7], which indicates that the number of MRONJ patients has increased with the increased number of systemic diseases requiring antiresorptive agents and/or other medications each year.

MRONJ worsens oral health-related quality of life [8], prevents ideal dental treatment, and decreases activities of daily living, due to chewing disability and defects of jaw bones caused by surgical treatment and/or pathological progression of MRONJ. Therefore, clarifying the exact mechanisms of and establishing treatment strategies for MRONJ are imperative. However, scientific data about the pathophisiology of and definitive treatment strategies for MRONJ are limited. Indeed, sufficient scientific evidence for the current treatment methods has not been established, although several types of therapies for MRONJ have been tried. However, dentists have to perform daily dental treatment in patients who receive high-risk medications for developing MRONJ. Therefore, available clinical information about what we should do when performing dental treatments in osteoporosis and cancer patients receiving antiresorptive agents or other medications is imperative to prevent development of MRONJ. Thus, the aims of this study were to (1) comprehensively review the current definitions, epidemiology, risk factors, and staging of and treatment strategies for MRONJ; and (2) provide clinical considerations or recommendations and precautions when dental treatment is performed in patients receiving high-risk medications for developing MRONJ to prevent its occurrence. The null hypothesis for this study was that "clinical considerations or recommendations for dental treatment are similar between in patients prior to or receiving high-risk medications for developing MRONJ and healthy patients not using these medications," even though this was a literature review without any statistical analysis and was not a systematic review and meta-analysis.

\section{Materials and methods PICO question}

The question in this literature review was formatted according to the Population, Intervention, Comparison, and Outcomes (PICO) framework, as follows:

- Population: Human subjects

- Intervention: Dental treatment in patients prior to or receiving high-risk medications for developing MRONJ

- Comparison: Dental treatment in healthy patients not taking high-risk medications for developing MRONJ

- Outcome: Clinical considerations or recommendations for dental treatment

\section{Literature search strategy}

A literature search was conducted on PubMed. The following search terms were used: "osteonecrosis," "osteonecrosis of the jaw," "jaw necrosis," "bisphosphonate(s)," "denosumab," "RANKL," "medication," "antiresorptive(s), " "antiresorptive agent," "literature review," "review," "systematic review," "statement," and "guideline." The PubMed search was independently performed by the two reviewers (M.K. and S.K.). The inclusion criteria were as follows: (1) articles published in English or German from December 2014 to December 2020; (2) human studies; and (3) review articles, systematic reviews, systematic reviews and meta-analyses, consensus statements, and guidelines. The exclusion criteria were as follows: (1) animal studies; and (2) in vitro studies. The related entry key words were used in different 
combinations using the Boolean operators "AND" and "OR" for the PubMed search as follows: \#1 ("osteonecrosis" OR "osteonecrosis of the jaw" OR "jaw necrosis") AND \#2 ("bisphosphonate(s)" OR "denosumab" OR "RANKL" OR "medication" OR "antiresorptive" OR "antiresorptive agent") AND \#3 ("literature review" OR "review" OR "systematic review" OR "systematic review and meta-analysis" OR "statement" OR and "guideline"). Moreover, a manual search was also conducted in regard to all references from selected articles and other articles identified on the PubMed search, irrespective of publication date, and inclusion and exclusion criteria.

Studies were investigated by screening the titles and abstracts, and included in this study by final discussion between SK and MK. Additional reviewer (TS) was also consulted for agreement when 2 independent reviewers disagree the results of screening. A total of 161 articles that fulfilled the inclusion criteria on the PubMed search were selected. Seventy-five of the articles were systematic reviews with or without a meta-analysis, 2 articles were consensus statements, 5 articles were clinical guidelines, and 33 were other types of research articles. After title and abstract screening, a total of 22 articles were selected for full-text review in addition to 53 articles selected by manual search.

\section{Results}

\section{Current definition and staging of MRONJ}

First of all, a correct diagnosis of MRONJ is needed. Lesions in the maxillofacial region that fulfill the following clinical situations are diagnosed as MRONJ: "(1) exposed bone in the maxillofacial region that does not heal within 8 weeks after identification by a health care provider; (2) current or previous treatment with antiresorptive or antiangiogenic agents; and (3) no history of radiation therapy to the jaws or obvious metastatic disease to the jaws" $[1,2,9]$.

A well-established optimal staging system should be used after the diagnosis of MRONJ, since appropriate staging of MRONJ based on clinical symptoms and radiographic findings helps clinicians provide appropriate treatment strategies for MRONJ patients [1, 2, 10] (Tables 1 and 2), although there is no clinical evidence regarding which staging systems are recommended due to no validation of the different systems [10]. The setting of stage 0 has been controversial, since it has been reported that $50 \%$ of them developed MRONJ in clinical situations, although the remaining of $50 \%$ may not progress to clinically evident MRONJ [10, 11].

\section{Epidemiology of MRONJ}

Recently, evidence regarding the epidemiology of MRONJ has been accumulated. In this section, the prevalence and incidence rates of BRONJ, DRONJ, and other MRONJs are described by the type of drug administered (Table 3).

\section{High-dose bisphosphonate and denosumab users with cancers}

The latest systematic review and meta-analysis with 13 , 857 patients reported that the prevalence of BRONJ in cancer patients receiving zoledronic acid (Zol) ranged from $0.4 \%$ to $1.6 \%, 0.8 \%$ to $2.1 \%$, and $1.0 \%$ to $2.3 \%$ after 1,2 , and 3 years of Zol exposure, respectively, whereas the prevalence of DRONJ in cancer patients receiving denosumab ranged from $0.5 \%$ to $2.1 \%, 1.1 \%$ to $3.0 \%$, and $1.3 \%$ to $3.2 \%$ after 1,2 , and 3 years of denosumab exposure, respectively [12]. The study also showed that DRONJ occurred significantly more often than BRONJ

Table 1 Clinical and imaging findings of MRONJ in each stage

\begin{tabular}{|c|c|c|}
\hline Staging & Clinical symptoms & Imaging findings \\
\hline Stage 0 & $\begin{array}{l}\text { no bone exposure/necrosis, deep periodontal pocket, loose tooth, } \\
\text { oral mucosal ulcer, swelling, abscess formation, trismus, } \\
\text { hypoesthesia/numbness of the lower lip (Vincent's symptom), non- } \\
\text { odontogenic pain }\end{array}$ & \multirow{3}{*}{$\begin{array}{l}\text { sclerotic alveolar bone, remaining tooth extraction socket, alveolar } \\
\text { bone loss or resorption not attributable to chronic periodontal } \\
\text { disease, changes to trabecular pattern-dense bone and no new } \\
\text { bone in extraction sockets, regions of osteosclerosis involving the } \\
\text { alveolar bone or surrounding basilar bone, thickening or obscuring } \\
\text { of the periodontal ligament (thickening of the lamina dura, sclerosis, } \\
\text { and decreased periodontal ligament space) }\end{array}$} \\
\hline Stage 1 & $\begin{array}{l}\text { asymptomatic bone exposure/necrosis without signs of infection, or } \\
\text { fistula in which the bone is palpable with a probe }\end{array}$ & \\
\hline Stage 2 & $\begin{array}{l}\text { bone exposure/necrosis associated with pain, infection, fistula in } \\
\text { which bone is palpable with a probe or at least one of the following } \\
\text { symptoms including bone exposure/necrosis over the alveolar bone, } \\
\text { which result in pathologic fracture, extraoral fistula, nasal/maxillary } \\
\text { sinus fistula formation, or advanced osteolysis extending to the } \\
\text { mandibular inferior edge or maxillary sinus }\end{array}$ & \\
\hline Stage 3 & $\begin{array}{l}\text { bone exposure/necrosis associated with pain, infection, or at least } \\
\text { one of the following symptoms, or fistula in which bone is palpable } \\
\text { with a probe. Bone exposure/necrosis over the alveolar bone. As a } \\
\text { result, pathologic fracture or extraoral fistula, nasal/maxillary sinus } \\
\text { fistula formation, or advanced osteolysis extending to the } \\
\text { mandibular inferior edge or maxillary sinus }\end{array}$ & $\begin{array}{l}\text { osteosclerosis/osteolysis of the surrounding bone (cheek bone, } \\
\text { palatine bone), pathologic mandibular fracture, and osteolysis } \\
\text { extending to the maxillary sinus floor }\end{array}$ \\
\hline
\end{tabular}


Table 2 Staging and treatment strategies for MRONJ

\begin{tabular}{ll}
\hline Staging & Clinical symptoms \\
\hline Stage 0 & $\begin{array}{l}\text { systemic management, including use of pain medications and antibiotics improvement of oral hygiene (rinsing and cleaning of fistulas and } \\
\text { periodontal pockets) }\end{array}$ \\
Stage 1 & $\begin{array}{l}\text { antibacterial mouth rinse clinical follow-up on a quarterly basis improvement of oral hygiene (rinsing and cleaning of fistulas and } \\
\text { periodontal pockets) patient education and review of indications for continued bisphosphonate therapy }\end{array}$ \\
Stage 2 & $\begin{array}{l}\text { combination or monotherapy of symptomatic treatment with oral antibiotics and/or oral antibacterial mouth rinse pain control } \\
\text { debridement to relieve soft tissue irritation and infection control }\end{array}$ \\
Stage 3 & $\begin{array}{l}\text { antibacterial mouth rinse antibiotic therapy and pain control surgical debridement or resection for longer-term palliation of infection and } \\
\text { pain extraction of tooth in exposed bone/necrotic bone as source of infection maintenance of nutrition with supplements and infusions }\end{array}$ \\
\hline
\end{tabular}

in cancer patients after 1,2 , and 3 years of exposure to antiresorptive agents, although the prognosis of DRONJ was similar to that of BRONJ [12]. The International Task Force on Osteonecrosis of the Jaw has reported that the incidence of BRONJ in cancer patients treated with intravenous (IV) bisphosphonates including Zol ranged from 0 to 12,222 per 100,000 patient-years, whereas the incidence of DRONJ in cancer patients treated with denosumab ranged from 0 to 2,316 per 100 , 000 patient-years [2].

\section{Low-dose bisphosphonate and denosumab users with osteoporosis}

It has been estimated that the prevalence of antiresorptive agent-related ONJ (ARONJ) in osteoporosis patients ranges from 0.001 to $0.01 \%$ in Australia, Canada, Germany, the USA, and Sweden. It has been reported that the prevalence of BRONJ in osteoporosis patients taking oral bisphosphonates ranges $0 \%$ to $0.04 \%$, although it is usually less than $0.001 \%$ [2, 9, 13-15]. However, there have been limited data on the prevalence of DRONJ.

The incidence of BRONJ in patients taking oral bisphosphonates for the treatment of osteoporosis ranges from 1.04 to 69 per 100,000 patient-years, whereas in osteoporosis patients receiving IV bisphosphonates, it ranges from 0 to 90 per 100,000 patientyears. On the other hand, the incidence of DRONJ in patients receiving denosumab for treatment of osteoporosis ranges from 0 to 30.2 per 100,000 patient-years. Therefore, the incidence of ARONJ in osteoporosis patients is relatively very low, regardless of the types of antiresorptive agents [2].

\section{Angiogenic inhibitor users}

Guarneri et al have reported that the prevalence of ONJ in advanced breast cancer patients receiving an angiogenic inhibitor, monoclonal antibody to VEGF (bevacizumab), ranged from 0.3 to $0.4 \%$, whereas the prevalence with bevacizumab and bisphosphonate combination therapy ranged from 0.9 to $2.4 \%$ [16].

\section{Other medication users}

One systematic review and one review have reported on MRONJ unrelated to antiresorptive agents and angiogenic inhibitors [7, 17], although there were not descriptions regarding the incidence and prevalence of MRONJ unrelated to them. In 2019, 30 tyrosine kinase inhibitors were approved. Between 2010 and 2014, 418 cases of ONJ induced by tyrosine kinase inhibitors (sunitinib, sorafenib, pazopanib, and axitinib) were reported to the U.S. Food and Drug Administration [18]. Other tyrosine kinase inhibitors such as imatinib [19], regorafenib [20], and cabozantanib [21] have also been reported to possibly cause MRONJ. Monoclonal antibodies (rituximab, adalimumab, infliximab, and romosozumab) have also

Table 3 Epidemiology of MRONJ

\begin{tabular}{|c|c|c|}
\hline MRONJ-inducing drugs & Prevalence & Incidence \\
\hline $\begin{array}{l}\text { Oral bisphosphonates Intravenous } \\
\text { bisphosphonates }\end{array}$ & $\begin{array}{l}<0.001 \% \text { (0\% to } 0.04 \%) 1.0 \% \text { to } 2.3 \% \text { for } 3 \text {-year } \\
\text { administration }\end{array}$ & $\begin{array}{l}1.04 \text { to } 69 \text { per } 100,000 \text { patient-years } 0 \text { to } 12,222 \text { per } \\
100,000 \text { patient-years }\end{array}$ \\
\hline Anti-RANKL antibody Osteoporosis Cancer & $1.3 \%$ to $3.2 \%$ for 3 -year administration & $\begin{array}{l}0 \text { to } 30.2 \text { per } 100,000 \text { patient-years } 0 \text { to } 2,316 \text { per } \\
100,000 \text { patient-years }\end{array}$ \\
\hline Angiogenic inhibitors & $0.3 \%$ to $0.4 \%$ & \\
\hline Anti-sclerostin antibody & Unknown & Unknown \\
\hline \multicolumn{3}{|l|}{ Anti-TNFa antibody } \\
\hline \multicolumn{3}{|l|}{ Anti-CD20 antibody } \\
\hline \multicolumn{3}{|l|}{ Other monoclonal antibodies } \\
\hline \multicolumn{3}{|l|}{ Tyrosine kinase inhibitors } \\
\hline mTOR inhibitors & & \\
\hline
\end{tabular}


been reported to possibly induce MRONJ, as well as denosumab and bevacizumab. Two cases by rituximab [22, 23], 4 cases by adalimumab and infliximab, which are monoclonal TNF- $\alpha$ antibodies [24], and 2 cases by romosozumab, which is a human monoclonal sclerostin antibody [25], have been reported to possibly cause MRONJ. mTOR inhibitors (everolimus and temsirolimus) and immunosuppressants (methotrexate and corticosteroids) may also cause MRONJ. However, caution is needed when interpreting MRONJ cases unrelated antiresorptive drugs and angiogenic inhibitors, since MRONJ cases caused by the above-mentioned medications are extremely rare $[7,17]$.

\section{Triggering factors and risk factors for MRONJ, comorbidities, and medications Triggering factors for MRONJ}

A recent systematic review investigating 3198 cases of BRONJ reported the triggering factors for BRONJ, regardless of administration routes, with $61.7 \%, 14.8 \%$, $7.4 \%, 7.2 \%, 5.0 \%$, and $3.9 \%$ of BRONJ triggered by tooth extraction, spontaneous onset, prosthesis-induced trauma such as ill-fitting dentures, history of dental surgery, periodontitis, and dental implant-related treatment, respectively [26].

On the contrary, there is limited information about the triggering factors for DRONJ. Two studies have reported that tooth extraction, poor oral hygiene, or dental appliances such as removable prostheses are among potential triggering factors for DRONJ [27, 28]. Currently, no clinical information on the triggering factors for MRONJ unrelated to antiresorptive agents is available.

\section{Risk factors for MRONJ}

Based on the recent review and systematic review of the risk factors for MRONJ, statements of the International Task Force on ONJ, the Position paper on ONJ of the American Association of Oral and Maxillofacial Surgeons (AAOMS), and the Position paper 2017 of the Japanese Allied Committee on ONJ [1, 2, 9, 29, 30], the following local risk factors have been identified. As mentioned above, IV and oral bisphosphonates and denosumab are undoubtedly risk factors for developing MRONJ $[1,2,9,29,30]$. Moreover, angiogenesis inhibitors, tyrosine kinase inhibitors, monoclonal antibodies, mTOR inhibitors, and immunosuppressants have been reported as possible risk factors for the development of MRONJ [7, 16, 17, 24] (Table 4).

\section{Local risk factors}

Tooth extraction is the most often reported risk factor for MRONJ. MRONJ occurs more commonly in the mandible than in the maxilla (65\% vs. $28.4 \%$ in the mandible vs. maxilla, $6.5 \%$ in both), although reasonable biological mechanisms remain unknown. Moreover, periodontal disease, acute dental infection, dental implant treatment (implant placement, bone augmentation, peri-implantitis, and removal), periodontal surgery, other oral surgeries, endodontic treatment, removable and fixed dental prostheses, trauma induced by ill-fitting dentures, anatomical factors (maxillary and mandibular tori, exostoses, knife-edge ridge, and mylohyoid ridge), and other oral conditions (excessive bite force, poor oral hygiene, and xerostomia) have also been implicated [ 1 , 2, 9, 29-31].

\section{Systemic risk factors}

Chemotherapy for malignant tumors (multiple myeloma, and breast, prostate, lung, renal and colon cancers), corticosteroid use, diabetes, tobacco use, and cardiovascular disease (hypertension, hyperlipidemia, and angina) are the most commonly reported systemic risk factors. Oncology patients receiving IV bisphosphonates or highdose denosumab are at risk for the development of MRONJ. Osteoporosis, rheumatoid arthritis, Sjögren's syndrome, sarcoidosis, hypocalcemia, hypoparathyroidism, osteomalacia, vitamin D deficiency, renal dialysis, anemia, Paget's disease of bone, erythropoietin therapy, cyclophosphamide therapy, alcohol intake, and obesity have also been reported to be systemic risk factors for developing MRONJ [1, 2, 9, 29, 30].

In a study of 2674 patients diagnosed with BRONJ, they had the following comorbidities and medications: chemotherapy (39.7\%), corticosteroid therapy (24.6\%), diabetes mellitus (11.2\%): hypertension (8.0\%), smoking habit $(8.0 \%)$, thrombin coagulopathies $(4.0 \%)$, and no concomitant diseases (4.1\%) [26]. On the other hand, denosumab-treated patients diagnosed with DRONJ have been reported to receive chemotherapy (64\% [27] and 75\% [28] of patients with DRONJ) or angiogenic inhibitors (20\% of patients with DRONJ) [28].

It has also been well documented that advanced age is one of the significant risk factors for developing BRONJ in bisphosphonate users, regardless of administration route $[2,32,33]$. A recent report showed that MRONJ occurred most commonly in older patients (median age 60 years) [17]. Therefore, caution should be exercised when dental treatment is performed in patients more than 60 years old.

\section{Other risk factors}

It has been reported that genetic factors such as singlenucleotide polymorphisms (SNPs; CYP2C8 and SIRT1/ HERC4) may become risk factors for MRONJ, although a recent systematic review concluded that SNPs were not correlated with the developing MRONJ [34]. Root canal treatment and orthodontic treatment are not risk factors for MRONJ [30]. 
Table 4 Risk factors for MRONJ

\begin{tabular}{|c|c|c|}
\hline \multicolumn{3}{|l|}{ Risk factors } \\
\hline \multirow{4}{*}{$\begin{array}{l}\text { Local risk } \\
\text { factors }\end{array}$} & Anatomical factors & mandible rather than maxilla maxillary or mandibular tori exostoses knife-edge ridgemylohyoid ridge \\
\hline & Dental treatment & $\begin{array}{l}\text { tooth extraction implant treatment (placement, bone augmentation, periimplantitis, removal) periodontal surgery } \\
\text { endodontic treatment (especially apicectomy) other oral surgery except for above-mentioned risk factors }\end{array}$ \\
\hline & Dental prosthesis & fixed prostheses (non-passive fit) ill-fitting dentures \\
\hline & $\begin{array}{l}\text { Other oral } \\
\text { conditions }\end{array}$ & excessive bite force poor oral hygiene xerostomia \\
\hline \multirow{3}{*}{$\begin{array}{l}\text { Systemic risk } \\
\text { factors }\end{array}$} & Medications & chemotherapy for malignant tumors corticosteroids \\
\hline & Systemic diseases & $\begin{array}{l}\text { oncology patients receiving IV bisphosphonates or high-dose denosumab diabetes osteoporosis rheumatoid } \\
\text { arthritis cardiovascular disease (hypertension, hyperlipidemia and angina) Sjögren's syndrome sarcoidosis } \\
\text { hypocalcemia hypoparathyroidism osteomalacia vitamin D deficiency renal dialysis anemia Paget's disease of bone }\end{array}$ \\
\hline & Others & tobacco use alcohol intake obesity advanced age \\
\hline $\begin{array}{l}\text { Other risk } \\
\text { factors }\end{array}$ & Genetic factors & single-nucleotide polymorphisms (CYP2C8, SIRT1/HERC4) \\
\hline
\end{tabular}

Clinical considerations in dental treatment for the prevention of MRONJ

Cancer patients prior to initiation of high-dose

antiresorptive agents $[1,9,10,30,35]$

It has been well documented that cancer patients receiving powerful antiresorptive therapy are among those at highest risk for the development of BRONJ/DRONJ. Therefore, if the patient's systemic condition permits, prior to initiation of powerful antiresorptive therapy, a good oral condition should be provided by comprehensive dental assessment including radiographic dental, periodontal, and radiographic examinations. Patient education for the maintenance of good oral hygiene and regarding the risk factors for the development of ARONJ is also important. Appropriate management of patients prior to high-dose antiresorptive therapy decreases the incidence of BRONJ/DRONJ [35-38].

Unrestorable and/or poor prognosis teeth should be extracted. Other necessary elective dentoalveolar surgery should also be completed. Antiresorptive therapy may be started after epithelial wound coverage is completed if the systemic condition permits. In humans, epithelial wound coverage is complete in 14 to 21 days. In addition to denture-induced ulcers on mucosa, especially the areas around the mylohyoid line and the maxillary or mandibular torus, the fitting of complete dentures and removable partial dentures should be examined when patients use either or both of them. Their relining or new fabrication should be performed prior to initiation of antiresorptive therapy when the dentures are clinically judged as ill-fitting. Ideally, placement of dental implants should be completed before the administration of high-dose antiresorptive agents, although nonurgent surgical procedures should be delayed if necessary [2, 9]. Cessation of smoking is strongly recommended. Uncontrolled systemic diseases that are risk factors for developing BRONJ/DRONJ should be under control, if possible, prior to initiation of antiresorptive therapy. The start date of antiresorptive therapy must be decided by the oncologist or physician after dentists communicate fully with them regarding dental treatment. Both physicians and dentists must explain the benefits of antiresorptive therapies and the risks of BRONJ/DRONJ to patients before anti-resorptive therapy. However, high-dose antiresorptive therapy may be acceptable in parallel with dental education, examination, and treatment when high-dose antiresorptive therapy cannot be delayed due to progression of bone metastasis and the need for therapy to inhibit SREs [30].

\section{Osteoporosis patients prior to initiation of antiresorptive agents [1, 9, 10, 30, 35]}

Osteoporosis is well known as one of the risk factors for developing BRONJ/DRONJ, although the incidence of $\mathrm{BRONJ/DRONJ}$ is relatively low in oral bisphosphonate or denosumab users for osteoporosis treatment compared to the incidence in high-dose IV bisphosphonate or denosumab users with malignant tumors. Clinical considerations for MRONJ in osteoporosis patients taking oral bisphosphonates and subcutaneous denosumab are basically the same for those in cancer patients receiving powerful antiresorptive agents. In particular, dentists should educate patients that the risk of developing BRONJ seems to increase when administration duration exceeds 3 or 4 years in oral bisphosphonate users [1, 39], since long-term treatment periods are generally required for osteoporosis. The same point should be made to denosumab users for osteoporosis, although there is limited information on the relationship between administration duration of denosumab and the risk of developing DRONJ. 


\section{Cancer and osteoporosis patients receiving antiresorptive therapy}

To suppress and decrease the potential risk of the development of ARONJ, some clinical recommendations to prevent ARONJ have been reported in position papers, guideline, consensus statements, or reviews $[1,2,10,30$, $35,40]$. In this section, some clinical protocols for the prevention of ARONJ are introduced, although all of them do not always have high-level scientific evidence. First of all, maintaining good oral hygiene and dental care is the most important measure to prevent new pathological lesions possibly triggering ARONJ. Dental education should also be continued.

Surgical treatment including tooth extraction in antiresorptive agent users Tooth extraction has been well documented to be one of the most serious risk factors for developing ARONJ [1, 2, 10, 30, 35]. The estimated risk for developing BRONJ after tooth extraction in cancer patients receiving IV bisphosphonates ranges from 1.6 to $14.8 \%[1,10]$. Most recently, it has been recommended that dentists start invasive dental treatment without discontinuation of IV or oral bisphosphonates $[10,30]$, although it has been previously recommended that non-restorable teeth may be treated by removal of the crown and endodontic treatment of the remaining roots without extraction in cancer patients receiving IV bisphosphonates [1]. Perioperative administration of antibacterial agents is recommended when tooth extraction is performed. Moreover, it has been recommended that smoothing the remaining sharp edges of alveolar bone and wound closure with mucoperiosteal flaps lined by periosteum should be performed [30]. On the other hand, there is limited information about the relationship between tooth extraction and denosumab users. However, a recent clinical guideline and the Japanese position paper have recommended that invasive dental treatment including tooth extraction could be performed without a drug holiday [10, 30].

Dental implant treatment in antiresorptive agent users Placement of dental implants, removal of dental implants, peri-implantitis, and taking oral bisphosphonates after implant placement have been reported to trigger the induction of ARONJ in osteoporosis or cancer patients [30, 31, 41-44]. The differences and the degrees of risk for developing ARONJ among these implant-related events have not been fully elucidated, since scientific and clinical data are limited. However, based on our current knowledge, administration route (oral vs. intravenous), dose (low-dose vs. high-dose), duration (short-term vs. long-term), and surgical site (maxillae vs. mandible, or anterior vs. posterior sites) may be associated with the development of ARONJ triggered by implant-related events. Implant removal should not be considered in cancer or osteoporosis patients receiving oral and/or intravenous bisphosphonates with peri-implantitis, since implant removal may possibly be one of the risk factors for developing ARONJ [31]. Strict maintenance programs such as short-term recall intervals, oral examinations of soft and hard tissues around natural teeth and dental implants, radiographical evaluations, and professional cleaning of peri-implant tissue are highly recommended.

The AAOMS has proposed that the risk for developing ARONJ in implant treatment is considered to be comparable to that in tooth extraction [1]. Implant-related surgical procedures are contraindicated in cancer patients receiving high-dose antiresorptive agents. On the other hand, implant treatment may be allowed without a drug holiday in osteoporosis patients receiving oral bisphosphonates or denosumab [1, 9, 30, 35]. In particular, the AAOMS has recommended that clinical information about possible implant failure with the risk for developing BRONJ should be provided when implant treatment is performed in osteoporosis patients receiving oral bisphosphonates for less than 4 years with no other medical risk factors, although the risks of implant failure and development of ARONJ are relatively low [1, 35]. On the contrary, there are no scientific data for clinical recommendations about implant treatment in osteoporosis patients taking oral bisphosphonates for less than 4 years with other medical risk factors and for more than 4 years with or without any medical risk factors [1]. There is a lack of information about whether implant treatment could be performed. Therefore, caution should be exercised when dental implant treatment is planned for osteoporosis patients taking oral bisphosphonates for more than 4 years, for less than 4 years with medical risk factors, or for osteoporosis patients receiving denosumab, regardless of administration period.

Periodontal therapy in antiresorptive agent users Periodontal disease has been well documented as a risk factor for the development of ARONJ [1, 2, 9, 10, 26, 30]. Recently, a systematic review and meta-analysis has also shown that periodontitis poses a higher risk for developing MRONJ, with a risk ratio of 2.75 (95\% confidence interval: 1.67-4.52) [45]. Thus, periodontal treatment is required for patients receiving antiresorptive agents to prevent and decrease the risk of ARONJ. However, periodontal surgery has been reported to be considered a risk factor for developing ARONJ, similar to tooth extraction [1]. Non-surgical treatment including pocket probing and scaling and root planning for periodontal disease is recommended. 
Prosthetic treatment in antiresorptive agent users There has been clinical evidence that wearing of illfitting dentures is one of the risk factors for the development of ARONJ [1, 2, 9, 10, 30, 35]. Therefore, removable partial and full dentures should be examined and evaluated when patients receiving antiresorptive agents visit dental clinics, even when patients have no chief complaint related to wearing dentures [1]. New fabrication or relining of dentures should be performed for denture stability [1,35]. Fixed partial dentures or single crowns with precise margins may be fabricated, although whether they are the risk factors for developing ARONJ remains unclear [46].

Endodontic treatment in antiresorptive agent users Endodontic treatment is not considered to be a risk factor for developing ARONJ [30]. However, apicoectomy has been considered a risk factor for developing ARONJ with the same level of risk as tooth extraction [1]. Moreover, periapical lesions have also been considered to be risk factors for the development of ARONJ [1, 47]. Therefore, to resolve periapical lesions, root canal treatment may proceed to prevent the development of ARONJ in antiresorptive users, although apicectomy should be avoided [35].

Orthodontic treatment in antiresorptive agent users There has been no evidence to establish that orthodontic treatment is a risk factor for developing ARONJ. Therefore, in current clinical situations, orthodontic treatment is not considered to be a risk factor for developing ARONJ [30]. Dentists can start orthodontic treatment in antiresorptive agent users, although it is difficult to move teeth ideally due to the effects of antiresorptive drugs. However, caution should be taken when patients receiving antiresorptive drugs, for whom orthodontic treatment is planned to start, have other risk factors for the development of ARONJ.

\section{Patients prior to initiation of or receiving other medications for developing MRONJ}

There have been no evidence-based clinical recommendations for the prevention of MRONJ induced by medications other than antiresorptive drugs. Basically, clinical recommendations for prevention of MRONJ may follow those in antiresorptive users proposed by the position papers, guidelines, consensus statements, or reviews $[1$, $2,10,30,35,40]$. More clinical evidences is needed to establish preventive protocols for MRONJ.

\section{Treatment strategies for MRONJ}

There is limited evidence regarding the mechanisms of MRONJ, although several animal MRONJ models have been developed to address them [48-58]. Therefore, current treatment strategies for MRONJ have been constructed based on clinical aspects rather than scientific data. In this section, the treatment strategy for MRONJ at each stage is introduced based on several position papers $[1,14,30,35]$, a clinical guideline [10], and other studies including systematic reviews and consensus statements by the International Task Force on ONJ [2, 9, 26, 41, 59].

\section{At risk}

No treatment is needed for the "At risk" stage, which is characterized by patients who have or had been treated with IV or oral antiresorptive or antiangiogenic therapy. However, patient education regarding MRONJ (prevalence, risks, and symptoms), maintenance of good oral hygiene, and reduction of modifiable risk factors is required to avoid development of MRONJ [1, 10, 26, 35, 59].

\section{Stage 0}

It has been reported that $50 \%$ of patients in "Stage 0" have progressed to stage 1, 2, or 3 [11]. Some reports have discussed Stage 0 with BRONJ patients [11, 60, 61]. The AAOMS [1], the Multinational Association of Supportive Care in Cancer/International Society of Oral Oncology (MASCC/ISOO) and the American Society of Clinical Oncology (ACSO) [10], the Japanese Allied Committee on ONJ [30], and the Korean Society for Bone and Mineral Research and the Korean Association of Oral and Maxillofacial Surgeons have included Stage 0 in staging [35], although the International Task Force on ONJ has not included it in the staging of MRONJ due to the potential risk of overdiagnosis [2]. Therefore, caution should be taken when diagnosing patients who have some symptoms without exposed bone as Stage 0 MRONJ, due to overdiagnosis. However, symptomatic treatment (chronic pain and infection control by appropriate medications) and conservative management are recommended for patients with Stage 0. A close follow-up system should be created for the early detection of progression to a higher stage of MRONJ [1, 9, 10, 26, 30, 59].

\section{Stage 1}

Conservative therapy (e.g., the use of antimicrobial oral rinses, monitoring patients with Stage 1 four times a year, improvement of oral hygiene) is recommended along with continuing patient education. Communication between the oncologists and/or physicians and dentists is strongly encouraged. Immediate surgery is not required at this stage, although minor surgical procedures such as sequestration or removal of necrotic bone are recommended to reduce soft tissue trauma $[1,9,10,26,30,35]$.

\section{Stage 2}

Treatment strategies for MRONJ Stages 2 and 3 are difficult to distinguish clearly, since some position papers, 
clinical reviews, and a clinical guideline recommend conservative rather than surgical treatment. In the following sections, information about treatment strategies for MRONJ Stages 2 and 3 are provided based on position papers, a clinical guideline, and systematic reviews, without any personal opinions.

In addition to antimicrobial mouth rinses, systemic antimicrobial control is recommended with antibiotics, although there is limited clinical evidence regarding which antibiotics are better for patients with Stage 2 MRONJ [1, 9, 10, 26, 30, 35]. Pain control and removal or debridement of necrotic bone may be considered, since formation of a bacterial membrane on exposed bone has been reported to inhibit the efficacy of systemic antimicrobial therapy [62-64]. Follow-up every 8 weeks and patient education are also recommended based on the guideline for MRONJ [10].

\section{Stage 3}

As with other stages of MRONJ, treatment revolves around antimicrobial mouth rinse and systemic antibiotic therapy with pain control. However, the necessity for extensive treatment is greater in many MRONJ cases. Surgical removal of a superficial and well-defined sequestrum should be considered when adjacent soft tissue is irritated by the sequestrum. Moreover, resection of a sequestrum, partial resection of maxillary bone, or hemi-mandibular resection should be performed for longer-term symptom amelioration. Referral to surgeons and/or oncologists may be needed when performing surgical procedures, since the bone lesions that are planned for removal, debridement, or resection may include cancer metastasis $[1,9,10,26,30,35]$.

\section{Surgical treatment for MRONJ}

As already described, some position papers, clinical reviews, and a clinical guideline have recommended nonsurgical treatment strategies for amelioration and/or resolution of MRONJ [1, 9, 10, 26, 30, 35]. However, recently, a systematic review demonstrated that conservative and extensive surgical approaches improved the healing rate of MRONJ when compared with a nonsurgical approach [41]. The study reported that the healing rates of Stages 1, 2, and 3 with a nonsurgical approach were $33 \%, 24 \%$, and $0 \%$, respectively. On the other hand, the authors have demonstrated that the healing rates of MRONJ with conservative surgical and extensive surgical approaches were $72 \%$ and $87 \%$ for Stage 1, 79\% and $96 \%$ for Stage 2, and $27 \%$ and $81 \%$ for Stage 3, respectively. Therefore, a surgical approach, rather than a nonsurgical approach, may be considered first if the patient's systemic condition permits. However, the complete healing rate of MRONJ in cancer patients receiving high-dose antiresorptive therapy has been reported to be approximately $50 \%$, even though a surgical approach was chosen as a treatment strategy for MRONJ [65], which suggests that caution should be exercised, since a surgical approach is not always superior to a nonsurgical approach for treatment for MRONJ lesions.

\section{Cessation of antiresorptive drugs}

First of all, dentists should not decide the cessation of high-dose antiresorptive agents, known as a drug holiday, in the patients who have serious clinical conditions such as cancer or immunosuppression, since discontinuation of antiresorptive agents has a potential risk of the relapse of SREs or tumor induced hypercalcemia. There is limited evidence that cessation of antiresorptive agents contributes to preventing BRONJ/DRONJ in many position papers and a guideline $[1,9,10,30,35,38]$, although they have reported that cessation of antiresorptive agents is controversial.

The half-life of bisphosphonates has been reported to be more than 10 years due to the higher affinity to hydroxyapatite, although that of denosumab is about 26 days after administration [66]. Moreover, the European Calcified Tissue Society has concluded that denosumab should not be discontinued without consideration of alternative treatment [67].

On the other hand, many reports have assessed the effects of discontinuation of antiresorptive agents on the resolution and/or amelioration of BRONJ/DRONJ. Most studies have demonstrated no effects on the clinical outcomes by cessation of antiresorptive agents [68-73], whereas two of them reported that a drug holiday had a positive effect on healing of BRONJ/DRONJ $[74,75]$.

Therefore, theoretically, given the above-mentioned reasons, taking into account the risks and benefits of discontinuation of antiresorptive agents with respect to systemic conditions, cessation of antiresorptive agents for the prevention or healing/amelioration of BRONJ/ DRONJ is basically not recommended.

\section{Conclusions}

Within the limitation of this literature review due to no systematic review and meta-analysis, the current status of MRONJ, such as epidemiology, risk factors, and staging of and treatment strategies, was described based on the clinical guidelines for MRONJ, the position papers on MRONJ, consensus statements, and literature reviews. Some clinical recommendations for each dental treatment in osteoporosis patients and cancer patients prior to or currently receiving antiresorptive agents were also described. There is a certain level of clinical evidence supporting the clinical recommendations for bisphosphonate users, whereas there is limited evidence for denosumab, angiogenesis inhibitors, and other medications. The mode of action of each drug is quite different, 
although the clinical characteristics of MRONJ are similar among drugs. Therefore, caution should be exercised when dental treatment is performed for users of denosumab, angiogenesis inhibitors, and other medications. Further clinical evidence needs to be accumulated to adequately create clinical recommendations/guidelines for patients at higher risk of MRONJ.

\section{Abbreviations}

MRONJ: Medication-related osteonecrosis of the jaw;

BRONJ: Bisphosphonate-related osteonecrosis of the jaw;

DRONJ: Denosumab-related osteonecrosis of the jaw; ARONJ: Antiresorptive agent-related osteonecrosis of the jaw; SREs: Skeletal-related events; Zol: Zoledronic acid; VEGF: Vascular endothelial cell growth factor; TNF: Tumor necrosis factor; AAOMS: The American Association of Oral and Maxillofacial Surgeons

\section{Acknowledgements}

Not applicable.

\section{Authors' contributions}

SK and TS conceived and designed the study. MK and SK contributed to all reviews and interpretations for the selected articles. MK wrote the final paper, and SK was responsible for the English review of the manuscript. All authors approved the final version of the manuscript.

\section{Funding}

This work was supported by JSPS KAKENHI (Grant No. 18H02994).

\section{Availability of data and materials}

The datasets used and/or analyzed during the current study are available from the corresponding author on reasonable request.

\section{Declarations}

Ethics approval and consent to participate

Not applicable.

\section{Consent for publication}

Not applicable.

\section{Competing interests}

Mampei Kawahara, Shinichiro Kuroshima, and Takashi Sawase declare that they have no competing interests.

Received: 14 January 2021 Accepted: 17 March 2021

Published online: 14 May 2021

\section{References}

1. Ruggiero SL, Dodson TB, Fantasia J, Goodday R, Aghaloo T, Mehrotra B, et al. American Association of Oral and Maxillofacial Surgeons position paper on medication-related osteonecrosis of the jaw-2014 update. J Oral Maxillofac Surg. 2014;72(10):1938-56. https://doi.org/10.1016/j.joms.2 014.04.031.

2. Khan AA, Morrison A, Hanley DA, Felsenberg D, McCauley LK, O'Ryan F, et al. Diagnosis and management of osteonecrosis of the jaw: a systematic review and international consensus. J Bone Miner Res. 2015;30(1):3-23. https://doi.org/10.1002/jbmr.2405

3. Marx RE. Pamidronate (Aredia) and zoledronate (Zometa) induced avascular necrosis of the jaws: a growing epidemic. J Oral Maxillofac Surg. 2003;61(9): 1115-7. https://doi.org/10.1016/S0278-2391(03)00720-1

4. Aghaloo TL, Felsenfeld AL, Tetradis S. Osteonecrosis of the jaw in a patient on Denosumab. J Oral Maxillofac Surg. 2010;68(5):959-63. https://doi.org/1 0.1016/j.joms.2009.10.010.

5. Taylor KH, Middlefell LS, Mizen KD. Osteonecrosis of the jaws induced by anti-RANK ligand therapy. Br J Oral Maxillofac Surg. 2010;48(3):221-3. https://doi.org/10.1016/j.bjoms.2009.08.030.
6. Estilo CL, Fornier M, Farooki A, Carlson D, Bohle G, Huryn JM. Osteonecrosis of the jaw related to bevacizumab. J Clin Oncol. 2008;26(24):4037-8. https:// doi.org/10.1200/JCO.2007.15.5424.

7. King R, Tanna N, Patel V. Medication-related osteonecrosis of the jaw unrelated to bisphosphonates and denosumab-a review. Oral Surg Oral Med Oral Pathol Oral Radiol. 2019;127(4):289-99. https://doi.org/10.1016/j. oooo.2018.11.012

8. Miksad RA, Lai KC, Dodson TB, Woo SB, Treister NS, Akinyemi O, et al. Quality of life implications of bisphosphonate-associated osteonecrosis of the jaw. Oncologist. 2011;16(1):121-32. https://doi.org/10.1634/ theoncologist.2010-0183.

9. Khan AA, Morrison A, Kendler DL, Rizzoli R, Hanley DA, Felsenberg D, et al Case-Based Review of Osteonecrosis of the Jaw (ONJ) and Application of the International Recommendations for Management From the International Task Force on ONJ. J Clin Densitom. 2017;20(1):8-24. https:// doi.org/10.1016/j.jocd.2016.09.005.

10. Yarom N, Shapiro CL, Peterson DE, Van Poznak CH, Bohlke K, Ruggiero SL, et al. Medication-related osteonecrosis of the jaw: MASCC/ISOO/ASCO Clinical Practice Guideline. J Clin Oncol. 2019;37(25):2270-90. https://doi. org/10.1200/JCO.19.01186.

11. Fedele S, Porter SR, D'Aiuto F, Aljohani S, Vescovi P, Manfredi M, et al. Nonexposed variant of bisphosphonate-associated osteonecrosis of the jaw: a case series. Am J Med. 2010;123(11):1060-4. https://doi.org/10.1016/j.a mjmed.2010.04.033.

12. Limones A, Sáez-Alcaide LM, Díaz-Parreño SA, Helm A, Bornstein MM, Molinero-Mourelle P. Medication-related osteonecrosis of the jaws (MRONJ) in cancer patients treated with denosumab VS. zoledronic acid: a systematic review and meta-analysis. Med Oral Patol Oral Cir Bucal. 2020;25:e326-36. https://doi.org/10.4317/medoral.23324.

13. Ulmner M, Jarnbring F, Törring O. Osteonecrosis of the jaw in Sweden associated with the oral use of bisphosphonate. J Oral Maxillofac Surg. 2014 72(1):76-82. https://doi.org/10.1016/j.joms.2013.06.221.

14. Svejda B, Muschitz C, Gruber R, Brandtner C, Svejda C, Gasser RW, et al. [Position paper on medication-related osteonecrosis of the jaw (MRONJ)]. Wien Med Wochenschr. 2016;166(1-2):68-74. https://doi.org/10.1007/s103 54-016-0437-2.

15. Mavrokokki T, Cheng A, Stein B, Goss A. Nature and frequency of bisphosphonate-associated osteonecrosis of the jaws in Australia. J Oral Maxillofac Surg. 2007;65(3):415-23. https://doi.org/10.1016/j.joms.2006.10.061.

16. Guarneri V, Miles D, Robert N, Diéras V, Glaspy J, Smith I, et al. Bevacizumab and osteonecrosis of the jaw: incidence and association with bisphosphonate therapy in three large prospective trials in advanced breast cancer. Breast Cancer Res Treat. 2010;122(1):181-8. https://doi.org/10.1007/ s10549-010-0866-3.

17. Nicolatou-Galitis O, Kouri M, Papadopoulou E, Vardas E, Galiti D, Epstein JB, et al. Osteonecrosis of the jaw related to non-antiresorptive medications: a systematic review. Support Care Cancer. 2019;27(2):383-94. https://doi.org/1 0.1007/s00520-018-4501-x.

18. Zhang $X$, Hamadeh IS, Song S, Katz J, Moreb JS, Langaee TY, et al. Osteonecrosis of the Jaw in the United States Food and Drug Administration's Adverse Event Reporting System (FAERS). J Bone Miner Res. 2016;31(2):336-40. https://doi.org/10.1002/jbmr.2693.

19. Viviano M, Rossi M, Cocca S. A rare case of osteonecrosis of the jaw related to imatinib. J Korean Assoc Oral Maxillofac Surg. 2017;43(2):120-4. https:// doi.org/10.5125/jkaoms.2017.43.2.120.

20. Antonuzzo L, Lunghi A, Giommoni E, Brugia M, Di Costanzo F. Regorafenib also can cause osteonecrosis of the jaw. J Natl Cancer Inst. 2016;108(4): djw002. https://doi.org/10.1093/jnci/djw002.

21. Marino R, Orlandi F, Arecco F, Gandolfo S, Pentenero M. Osteonecrosis of the jaw in a patient receiving cabozantinib. Aust Dent J. 2015;60(4):528-31. https://doi.org/10.1111/adj.12254

22. Allegra A, Oteri G, Alonci A, Bacci F, Penna G, Minardi V, et al. Association of osteonecrosis of the jaws and POEMS syndrome in a patient assuming rituximab. J Craniomaxillofac Surg. 2014;42(4):279-82. https://doi.org/10.101 6/j.jcms.2013.05.014

23. Keribin P, Guerrot D, Jardin F, Moizan H. Osteonecrosis of the jaw in a patient presenting with post-transplantation lymphoproliferative disorder treated with rituximab: a case report. J Oral Maxillofac Surg. 2017;75(12): 2599-605. https://doi.org/10.1016/j.joms.2017.05.016.

24. Sacco R, Shah S, Leeson R, Moraschini V, de Almeida Barros Mourão CF, Akintola $\mathrm{O}$, et al. Osteonecrosis and osteomyelitis of the jaw associated with 
tumour necrosis factor-alpha (TNF-a) inhibitors: a systematic review. Br J Oral Maxillofac Surg. 2020;58:25-33. https://doi.org/10.1016/j.bjoms.2019.09. 023.

25. Cosman F, Crittenden DB, Adachi JD, Binkley N, Czerwinski E, Ferrari S, et al. Romosozumab treatment in postmenopausal women with osteoporosis. N Engl J Med. 2016;375(16):1532-43. https://doi.org/10.1056/NEJMoa1607948.

26. Fliefel R, Tröltzsch M, Kühnisch J, Ehrenfeld M, Otto S. Treatment strategies and outcomes of bisphosphonate-related osteonecrosis of the jaw (BRONJ) with characterization of patients: a systematic review. Int J Oral Maxillofac Surg. 2015;44(5):568-85. https://doi.org/10.1016/j.jom.2015.01.026.

27. Fizazi K, Carducci M, Smith M, Damião R, Brown J, Karsh L, et al. Denosumab versus zoledronic acid for treatment of bone metastases in men with castration-resistant prostate cancer: a randomised, double-blind study. Lancet. 2011;377(9768):813-22. https://doi.org/10.1016/S0140-6736(10)62344-6.

28. Stopeck AT, Lipton A, Body JJ, Steger GG, Tonkin K, de Boer RH, et al. Denosumab compared with zoledronic acid for the treatment of bone metastases in patients with advanced breast cancer: a randomized, doubleblind study. J Clin Oncol. 2010;28(35):5132-9. https://doi.org/10.1200/JCO.2 010.29.7101.

29. McGowan K, McGowan T, Ivanovski S. Risk factors for medication-related osteonecrosis of the jaws: A systematic review. Oral Dis. 2018;24(4):527-36. https://doi.org/10.1111/odi.12708

30. Yoneda T, Hagino H, Sugimoto T, Ohta H, Takahashi S, Soen S, et al. Antiresorptive agent-related osteonecrosis of the jaw: Position Paper 2017 of the Japanese Allied Committee on Osteonecrosis of the Jaw. J Bone Miner Metab. 2017;35(1):6-19. https://doi.org/10.1007/s00774-016-0810-7.

31. Yamamoto S, Maeda K, Kouchi I, Hirai Y, Taniike N, Yamashita D, et al. Development of antiresorptive agent-related osteonecrosis of the jaw after dental implant removal: a case report. J Oral Implantol. 2018;44(5):359-64. https://doi.org/10.1563/aaid-joi-D-18-00032.

32. Pazianas M, Miller P, Blumentals WA, Bernal M, Kothawala P. A review of the literature on osteonecrosis of the jaw in patients with osteoporosis treated with oral bisphosphonates: prevalence, risk factors, and clinical characteristics. Clin Ther. 2007;29(8):1548-58. https://doi.org/10.1016/j. clinthera.2007.08.008.

33. Badros A, Weikel D, Salama A, Goloubeva O, Schneider A, Rapoport A, et al. Osteonecrosis of the jaw in multiple myeloma patients: clinical features and risk factors. J Clin Oncol. 2006;24(6):945-52. https://doi.org/10.1200/JCO.2 005.04.2465

34. Sandro Pereira da Silva J, Pullano E, Raje NS, Troulis MJ, August M. Genetic predisposition for medication-related osteonecrosis of the jaws: a systematic review. Int J Oral Maxillofac Surg. 2019;48:1289-99. https://doi.org/10.1016/j. ijom.2019.04.014

35. Kim KM, Rhee Y, Kwon YD, Kwon TG, Lee JK, Kim DY. Medication related osteonecrosis of the jaw: 2015 Position Statement of the Korean Society for Bone and Mineral Research and the Korean Association of Oral and Maxillofacial Surgeons. J Bone Metab. 2015;22(4):151-65. https://doi.org/1 0.11005/jbm.2015.22.4.151.

36. Yamashita J, McCauley LK. Antiresorptives and osteonecrosis of the jaw. J Evid Based Dent Pract. 2012;12(3):233-47. https://doi.org/10.1016/S1532-33 82(12)70046-5.

37. Lo JC, O'Ryan F, Yang J, Hararah MK, Gonzalez JR, Gordon N, et al. Ora health considerations in older women receiving oral bisphosphonate therapy. J Am Geriatr Soc. 2011;59(5):916-22. https://doi.org/10.1111/j.15325415.2011.03371.x

38. Hellstein JW, Adler RA, Edwards B, Jacobsen PL, Kalmar JR, Koka S, et al. Managing the care of patients receiving antiresorptive therapy for prevention and treatment of osteoporosis: executive summary of recommendations from the American Dental Association Council on Scientific Affairs. J Am Dent Assoc. 2011;142(11):1243-51. https://doi.org/1 0.14219/jada.archive.2011.0108.

39. Stavropoulos A, Bertl K, Pietschmann P, Pandis N, Schiødt M, Klinge B. The effect of antiresorptive drugs on implant therapy: systematic review and meta-analysis. Clin Oral Implants Res. 2018;29(Suppl 18):54-92. https://doi. org/10.1111/clr.13282.

40. Romero-Ruiz MM, Romero-Serrano M, Serrano-González A, Serrera-Figallo MA, Gutiérrez-Pérez JL, Torres-Lagares D. Proposal for a preventive protocol for medication-related osteonecrosis of the jaw. Med Oral Patol Oral Cir Bucal. 2020:24197. https://doi.org/10.4317/medoral.24197.

41. Rupel K, Ottaviani G, Gobbo M, Contardo L, Tirelli G, Vescovi P, et al. A systematic review of therapeutical approaches in bisphosphonates-related osteonecrosis of the jaw (BRONJ). Oral Oncol. 2014;50(11):1049-57. https:// doi.org/10.1016/j.oraloncology.2014.08.016.

42. Schimmel M, Srinivasan M, McKenna G, Müller F. Effect of advanced age and/or systemic medical conditions on dental implant survival: a systematic review and meta-analysis. Clin Oral Implants Res. 2018;29(Suppl 16):311-30. https://doi.org/10.1111/clr.13288.

43. Kwon TG, Lee CO, Park JW, Choi SY, Rijal G, Shin HI. Osteonecrosis associated with dental implants in patients undergoing bisphosphonate treatment. Clin Oral Implants Res. 2014;25(5):632-40. https://doi.org/10.1111/clr.12088.

44. Troeltzsch M, Cagna D, Stähler P, Probst F, Kaeppler G, Ehrenfeld M, et al. Clinical features of peri-implant medication-related osteonecrosis of the jaw: Is there an association to peri-implantitis? J Craniomaxillofac Surg. 2016; 44(12):1945-51. https://doi.org/10.1016/j.jcms.2016.09.018.

45. Lorenzo-Pouso Al, Pérez-Sayáns M, Chamorro-Petronacci C, Gándara-Vila P, López-Jornet P, Carballo J, et al. Association between periodontitis and medication-related osteonecrosis of the jaw: A systematic review and metaanalysis. J Oral Pathol Med. 2020:49(3):190-200. https://doi.org/10.1111/jop.12963.

46. Niibe K, Ouchi T, Iwasaki R, Nakagawa T, Horie N. Osteonecrosis of the jaw in patients with dental prostheses being treated with bisphosphonates or denosumab. J Prosthodont Res. 2015;59(1):3-5. https://doi.org/10.1016/j. jpor.2014.08.001.

47. Marx RE, Sawatari Y, Fortin M, Broumand V. Bisphosphonate-induced exposed bone (osteonecrosis/osteopetrosis) of the jaws: risk factors, recognition, prevention, and treatment. J Oral Maxillofac Surg. 2005;63(11): 1567-75. https://doi.org/10.1016/j.joms.2005.07.010.

48. Williams DW, Lee C, Kim T, Yagita H, Wu H, Park S, et al. Impaired bone resorption and woven bone formation are associated with development of osteonecrosis of the jaw-like lesions by bisphosphonate and anti-receptor activator of NF-KB ligand antibody in mice. Am J Pathol. 2014;184(11):308493. https://doi.org/10.1016/j.ajpath.2014.07.010.

49. Pautke C, Kreutzer K, Weitz J, Knödler M, Münzel D, Wexel G, et al. Bisphosphonate related osteonecrosis of the jaw: a minipig large animal model. Bone. 2012;51(3):592-9. https://doi.org/10.1016/j.bone.2012.04.020.

50. Bi Y, Gao Y, Ehirchiou D, Cao C, Kikuiri T, Le A, et al. Bisphosphonates cause osteonecrosis of the jaw-like disease in mice. Am J Pathol. 2010;177(1):28090. https://doi.org/10.2353/ajpath.2010.090592.

51. López-Jornet P, Camacho-Alonso F, Molina-Miñano F, Gómez-García F, Vicente-Ortega $V$. An experimental study of bisphosphonate-induced jaws osteonecrosis in Sprague-Dawley rats. J Oral Pathol Med. 2010;39(9):697702. https://doi.org/10.1111/j.1600-0714.2010.00927.x.

52. Kuroshima S, Yamashita J. Chemotherapeutic and antiresorptive combination therapy suppressed lymphangiogenesis and induced osteonecrosis of the jaw-like lesions in mice. Bone. 2013;56(1):101-9. https:// doi.org/10.1016/j.bone.2013.05.013.

53. Kuroshima S, Sasaki M, Nakajima K, Tamaki S, Hayano H, Sawase T. Prevalence of bisphosphonate-related osteonecrosis of the jaw-like lesions is increased in a chemotherapeutic dose-dependent manner in mice. Bone. 2018;112:177-86. https://doi.org/10.1016/j.bone.2018.05.001.

54. Hayano H, Kuroshima S, Sasaki M, Tamaki S, Inoue M, Ishisaki A, et al. Distinct immunopathology in the early stages between different antiresorptives-related osteonecrosis of the jaw-like lesions in mice. Bone. 2020;135:115308. https://doi.org/10.1016/j.bone.2020.115308.

55. Allen MR, Chu TM, Ruggiero SL. Absence of exposed bone following dental extraction in beagle dogs treated with 9 months of high-dose zoledronic acid combined with dexamethasone. J Oral Maxillofac Surg. 2013;71(6): 1017-26. https://doi.org/10.1016/j.joms.2012.11.016.

56. de Molon RS, Shimamoto H, Bezouglaia O, Pirih FQ, Dry SM, Kostenuik P, et al. OPG-Fc but not zoledronic acid discontinuation reverses osteonecrosis of the jaws (ONJ) in mice. J Bone Miner Res. 2015;30(9):1627-40. https://doi. org/10.1002/jbmr.2490.

57. Soundia A, Hadaya D, Esfandi N, de Molon RS, Bezouglaia O, Dry SM, et al. Osteonecrosis of the jaws (ONJ) in mice after extraction of teeth with periradicular disease. Bone. 2016;90:133-41. https://doi.org/10.1016/j.bone.2 016.06.011.

58. Aghaloo TL, Cheong S, Bezouglaia O, Kostenuik P, Atti E, Dry SM, et al. RANK $L$ inhibitors induce osteonecrosis of the jaw in mice with periapical disease. J Bone Miner Res. 2014;29(4):843-54. https://doi.org/10.1002/jbmr.2097.

59. Moraschini V, Calasans-Maia MD, Louro RS, Arantes EBR, Calasans-Maia JA Weak evidence for the management of medication-related osteonecrosis of the jaw: An overview of systematic reviews and meta-analyses. J Oral Pathol Med. 2021;50:10-21. https://doi.org/10.1111/jop.13083. Epub 2020 Jul 12. 
60. O'Ryan FS, Khoury S, Liao W, Han MM, Hui RL, Baer D, et al. Intravenous bisphosphonate-related osteonecrosis of the jaw: bone scintigraphy as an early indicator. J Oral Maxillofac Surg. 2009;67(7):1363-72. https://doi.org/1 0.1016/j.joms.2009.03.005.

61. Schiodt M, Reibel J, Oturai P, Kofod T. Comparison of nonexposed and exposed bisphosphonate-induced osteonecrosis of the jaws: a retrospective analysis from the Copenhagen cohort and a proposal for an updated classification system. Oral Surg Oral Med Oral Pathol Oral Radiol. 2014; 117(2):204-13. https://doi.org/10.1016/j.0000.2013.10.010.

62. Kumar SK, Gorur A, Schaudinn C, Shuler CF, Costerton JW, Sedghizadeh PP. The role of microbial biofilms in osteonecrosis of the jaw associated with bisphosphonate therapy. Curr Osteoporos Rep. 2010;8(1):40-8. https://doi. org/10.1007/s11914-010-0008-1.

63. Sedghizadeh PP, Kumar SK, Gorur A, Schaudinn C, Shuler CF, Costerton JW. Identification of microbial biofilms in osteonecrosis of the jaws secondary to bisphosphonate therapy. J Oral Maxillofac Surg. 2008;66(4):767-75. https:// doi.org/10.1016/j.joms.2007.11.035.

64. Wanger G, Gorby Y, El-Naggar MY, Yuzvinsky TD, Schaudinn C, Gorur A, et al. Electrically conductive bacterial nanowires in bisphosphonate-related osteonecrosis of the jaw biofilms. Oral Surg Oral Med Oral Pathol Oral Radiol. 2013;115(1):71-8. https://doi.org/10.1016/j.000o.2012.08.446.

65. Hayashida S, Soutome S, Yanamoto S, Fujita S, Hasegawa T, Komori T, et al. Evaluation of the treatment strategies for medication-related osteonecrosis of the jaws (MRONJ) and the Factors affecting treatment outcome: a multicenter retrospective study with propensity score matching analysis. J Bone Miner Res. 2017;32(10):2022-9. https://doi.org/10.1002/jbmr.3191.

66. Baron R, Ferrari S, Russell RG. Denosumab and bisphosphonates: different mechanisms of action and effects. Bone. 201 1;48(4):677-92. https://doi.org/1 0.1016/j.bone.2010.11.020.

67. Tsourdi E, Langdahl B, Cohen-Solal M, Aubry-Rozier B, Eriksen EF, Guañabens $\mathrm{N}$, et al. Discontinuation of Denosumab therapy for osteoporosis: a systematic review and position statement by ECTS. Bone. 2017;105:11-7. https://doi.org/10.1016/j.bone.2017.08.003.

68. Hayashida S, Yanamoto S, Fujita S, Hasegawa T, Komori T, Kojima Y, et al. Drug holiday clinical relevance verification for antiresorptive agents in medication-related osteonecrosis cases of the jaw. J Bone Miner Metab. 2020;38(1):126-34. https://doi.org/10.1007/s00774-019-01035-7.

69. Hasegawa T, Hayashida S, Kondo E, Takeda Y, Miyamoto H, Kawaoka Y, et al. Medication-related osteonecrosis of the jaw after tooth extraction in cancer patients: a multicenter retrospective study. Osteoporos Int. 2019;30(1):231-9. https://doi.org/10.1007/s00198-018-4746-8.

70. Ottesen C, Schiodt M, Gotfredsen K. Efficacy of a high-dose antiresorptive drug holiday to reduce the risk of medication-related osteonecrosis of the jaw (MRONJ): a systematic review. Heliyon. 2020;6(4):e03795. https://doi. org/10.1016/j.heliyon.2020.e03795.

71. Nicolatou-Galitis O, Papadopoulou E, Sarri T, Boziari P, Karayianni A, Kyrtsonis MC, et al. Osteonecrosis of the jaw in oncology patients treated with bisphosphonates: prospective experience of a dental oncology referral center. Oral Surg Oral Med Oral Pathol Oral Radiol Endod. 2011;112(2):195202. https://doi.org/10.1016/.tripleo.2011.02.037.

72. Van den Wyngaert T, Claeys T, Huizing MT, Vermorken JB, Fossion E. Initial experience with conservative treatment in cancer patients with osteonecrosis of the jaw (ONJ) and predictors of outcome. Ann Oncol. 2009, 20(2):331-6. https://doi.org/10.1093/annonc/mdn630.

73. Bodem JP, Schaal C, Kargus S, Saure D, Mertens C, Engel M, et al. Surgical management of bisphosphonate-related osteonecrosis of the jaw stages II and III. Oral Surg Oral Med Oral Pathol Oral Radiol. 2016;121(4):367-72. https://doi.org/10.1016/j.0000.2015.10.033.

74. Martins AS, Correia JA, Salvado F, Caldas C, Santos N, Capelo A, et al. Relevant factors for treatment outcome and time to healing in medicationrelated osteonecrosis of the jaws-a retrospective cohort study. J Craniomaxillofac Surg. 2017;45(10):1736-42. https://doi.org/10.1016/j.jcms.2 017.07.014.

75. Wutzl A, Pohl S, Sulzbacher I, Seemann R, Lauer G, Ewers R, et al. Factors influencing surgical treatment of bisphosphonate-related osteonecrosis of the jaws. Head Neck. 2012;34(2):194-200. https://doi.org/10.1002/hed.21708.

\section{Publisher's Note}

Springer Nature remains neutral with regard to jurisdictional claims in published maps and institutional affiliations.

\section{Submit your manuscript to a SpringerOpen ${ }^{\circ}$ journal and benefit from:}

- Convenient online submission

- Rigorous peer review

- Open access: articles freely available online

- High visibility within the field

- Retaining the copyright to your article

Submit your next manuscript at $\boldsymbol{\nabla}$ springeropen.com 